OPEN ACCESS

Edited by:

Jean Mariani,

Université Pierre et Marie Curie,

France

Reviewed by:

Ricardo Osorio

New York University, United States

Arash Tadjalli,

University of Florida, United States

*Correspondence:

Mo Saffarini

journals@resurg.eu

Received: 26 November 2018

Accepted: 10 December 2019

Published: 10 January 2020

Citation:

Attier-Zmudka J, Sérot J-M

Valluy J, Saffarini M, Douadi Y, Malinowski KP and Balédent O (2020)

Sleep Apnea Syndrome in an Elderly

Population Admitted to a Geriatric Unit: Prevalence and Effect on Cognitive Function Front. Aging Neurosci. 11:361 doi: 10.3389/fnagi.2019.00361

\section{Sleep Apnea Syndrome in an Elderly Population Admitted to a Geriatric Unit: Prevalence and Effect on Cognitive Function}

\author{
Jadwiga Attier-Zmudka ${ }^{1,2}$, Jean-Marie Sérot ${ }^{1}$, Jeremy Valluy ${ }^{3}$, Mo Saffarini3*, \\ Youcef Douadi ${ }^{4}$, Krzysztof Piotr Malinowski ${ }^{5}$ and Olivier Balédent ${ }^{2,6}$
}

\begin{abstract}
'Department of Gerontology, Saint-Quentin Hospital, Saint-Quentin, France, ${ }^{2}$ CHIMERE, EA 7516 Head \& Neck Research Group, University of Picardy Jules Verne, Amiens, France, ${ }^{3}$ ReSurg SA, Nyon, Switzerland, ${ }^{4}$ Department of Pulmonology, Saint-Quentin Hospital, Saint-Quentin, France, ${ }^{5}$ Institute of Public Health, Faculty of Health Sciences, Jagiellonian University Medical College, Kraków, Poland, ${ }^{6}$ BioFlowlmage, Image Processing Unit, University Hospital of Amiens, Amiens, France
\end{abstract}

Background: Sleep apnea leads to cognitive impairment in older patients, but its association with neurodegeneration remains controversial, and most studies do not distinguish between the more common obstructive form (OSAS) and the rarer central form (CSAS).

Objective: The purpose of this study was to assess the prevalence of the different forms of sleep apnea in a cohort of cognitively impaired elderly patients (>70 years) and to investigate their associations with cognitive deficit, weighted against known risk factors for neurodegeneration.

Methods: Overnight polygraphy was performed for 76 consecutive patients admitted to our geriatric unit. Their cognitive function was assessed using the Mini Mental-State Exam (MMSE), Mattis Dementia Rating Scale (MDRS) and Stroop test. Multivariable analyses were performed to determine associations between cognitive function and independent variables describing demographics, sleep apnea measures, and cardiovascular risk factors.

Results: The cohort comprised 58 women and 18 men aged a mean of 84 years (range, 73-96). Sleep apnea syndrome (SAS) was diagnosed in 48 patients (63\%), of which 31 $(41 \%)$ with OSAS and 17 (22\%) with CSAS. Multivariable regression analysis revealed that MDRS was lower in patients with OSAS $(\beta=-10.03, p=0.018)$, that Stroop Colors and Words delays increased with $\mathrm{AHI}(\beta=0.17, p=0.030$ and $\beta=0.31, p=0.047)$ and that that Stroop Interference delay was higher in patients with CSAS ( $\beta=24.45$, $p=0.002$ ).

Conclusion: Sleep apnea is thus highly prevalent in elderly patients with cognitive impairment. OSAS was associated with lower general cognitive function, while CSAS was only associated with increased Stroop Interference delays. Elderly patients with cognitive deficit could benefit from sleep apnea screening and treatment.

\footnotetext{
Keywords: sleep apnea, cognitive deficit, elderly population, OSAS, CSAS
} 


\section{INTRODUCTION}

Sleep apnea syndrome (SAS) is a disorder characterized by repeated pauses in breathing during sleep (Young et al., 1993). It is often underdiagnosed (Peppard et al., 2013), though its prevalence is increasing and was recently shown to be up to $40 \%$ in the general population (Heinzer et al., 2015), and likely higher in the elderly population (Al Lawati et al., 2009; Heinzer et al., 2015). SAS interrupts sleep and causes intermittent hypoxia (Rosenzweig et al., 2015) which could contribute to a range of pathophysiological consequences, including cognitive impairment (Wallace and Bucks, 2013; Arli et al., 2015), particularly in older patients (Yaffe et al., 2011; Osorio et al., 2015; Tsapanou et al., 2018). In fact, SAS is more prevalent in patients suffering from neurodegenerative diseases (Moran et al., 2005; Emamian et al., 2016) and is suspected to increase risks of developing Alzheimer's disease (Aoki et al., 2014; Osorio et al., 2015; Liguori and Placidi, 2018).

The association between SAS and neurodegenerative diseases remains controversial (Somers et al., 2008; Rosenzweig et al., 2015) and the pathophysiological consequences of SAS are still unclear (Yaffe et al., 2011; Zimmerman and Aloia, 2012; Martin et al., 2015; Bubu et al., 2019). This is further complicated by the fact that most studies do not distinguish between the more common obstructive form (OSAS), characterized by recurrent collapse of the upper respiratory tract, and the rarer central form (CSAS), characterized by repetitive pauses in breathing without respiratory effort. Moreover, the causes of cognitive impairments are difficult to establish in patients over 70 years old, who often exhibit multiple comorbidities.

Validated modifiable risk factors can be used to mitigate the progression of age-related cognitive decline. For instance, OSAS is associated with Alzheimer's disease biomarker accumulation (Bubu et al., 2019) and can be treated with continuous positive airway pressure (CPAP) (Marshall et al., 2006; Cooke et al., 2009; Kylstra et al., 2013; Richards et al., 2019), which improves cognitive function in patients suffering from Alzheimer's disease, although its effect on pathological progression remains unknown (Ancoli-Israel et al., 2008; Troussiere et al., 2014; Dostalova et al., 2019). The purpose of this exploratory study was therefore to (a) assess the prevalence of each form of SAS in a cohort of elderly patients ( $>70$ years old) admitted to our geriatric unit for non-acute reasons, and (b) investigate the independent relation between sleep apnea and cognitive performance, measured using validated tests, including the Mini Mental-State Exam (MMSE), the Mattis Dementia rating Scale (MDRS) and the Stroop test, and competing against known risk factors for cognitive impairment. The hypothesis was that (i) the prevalence of SAS in elderly patients consulting for cognitive deficit would be greater than that reported for the general population and (ii) elderly patients with SAS would have significantly worse MMSE, MDRS and Stroop test scores, independently of confounding factors.

\section{MATERIALS AND METHODS}

We enrolled 115 consecutive patients admitted to our geriatric unit for non-acute reasons (non-emergency, no surgery, no mandatory overnight stay) between October 2015 and March 2018. The inclusion criterion was patients aged over 70 years. The exclusion criteria were (i) refusal to undergo sleep apnea diagnosis with overnight polygraph recording (26 patients), or (ii) refusal or inability to complete neurocognitive assessment using structural cerebral magnetic resonance imaging (MRI) (12 patients). In addition, 1 patient died before completing the cognitive assessment, which left a study cohort of 76 patients (Figure 1), who underwent overnight ambulatory polygraphy (Weinmann SOMNOlab 2, Hamburg, Germany) to measure their Apnea-Hypopnea Index (AHI), total number of events per night, mean arterial blood oxygen saturation $\left(\mathrm{SaO}_{2}\right)$ and sleep time at $\mathrm{SaO}_{2}$ below 90\% (Kuwabara et al., 2018). Apnea severity was noted based on the AHI, as mild (AHI 5-15), moderate (AHI 16-30), or severe (AHI > 30). Apneas were defined as cessation of airflow for at least $10 \mathrm{~s}$ and hypopneas were defined as a reduction of airflow to $\leq 50 \%$ of baseline for at least $10 \mathrm{~s}$ (Somers et al., 2008). The final diagnosis of sleep apnea, obstructive (OSAS) or central (CSAS) was determined by a pulmonologist (YD) based on the relative number of obstructive or central events per night, based on the definition by Somers et al. (2008): obstructive sleep apnea is characterized by ongoing ventilatory effort while central sleep apnea is characterized by withdrawal of central respiratory drive. OSAS and CSAS were defined when a patient had 5 or more episodes of obstructive or central apneas per hour of sleep, respectively.

The patient's overall cognitive function was assessed by clinical psychologists (IG and ASM) using the following validated scores: (a) Mini-Mental State Examination (MMSE) (Kalafat Michel et al., 2003), which ranges from 0 to 30 points. Patients scoring $\leq 23$ considered cognitively impaired. (b) MDRS, which comprises five subscales, spanning the areas of attention, initiation-perseveration, construction, conceptualization and

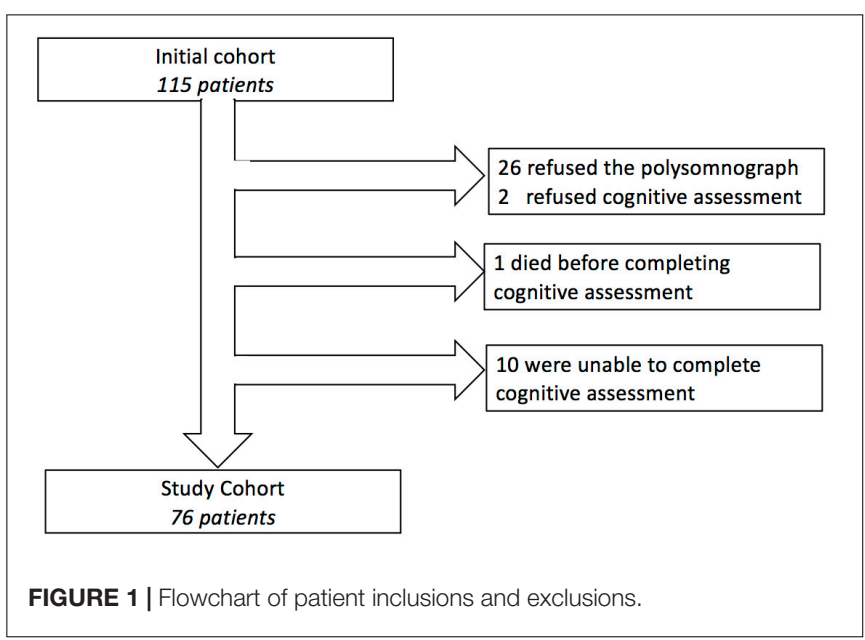


memory (Hersch, 1979; Gardner et al., 1981). (Heinzer et al., 2015) Stroop Colors and Words test was used to assess attention and executive functioning (Comalli et al., 1962), which are known to be affected by sleep apnea (Olaithe and Bucks, 2013; Martin et al., 2015; Rosenzweig et al., 2015). The Colors test asks patients to recognize the color of dots that appear for a short time, while the Words test asks them to read words (names of colors) that appear for a short time. The Interference test requires the patients to read the words (names of colors)

TABLE 1 | Patient demographics.

\begin{tabular}{|c|c|c|c|}
\hline & \multicolumn{3}{|c|}{ Cohort $(n=76)$} \\
\hline & Median & [IQR] & (Range) \\
\hline Age (years) & 84 & {$[80-88]$} & $(73-96)$ \\
\hline $\mathrm{BMI}\left(\mathrm{kg} / \mathrm{m}^{2}\right)$ & 24 & {$[21-27]$} & $(16-40)$ \\
\hline Depression (MADRS*/60) & 6 & {$[3-11]$} & $(0-31)$ \\
\hline \multirow[t]{2}{*}{ Anxiety (Goldberg/9) } & 3 & {$[1-5]$} & $(0-9)$ \\
\hline & $n$ & $(\%)$ & \\
\hline Women & 58 & $(76 \%)$ & \\
\hline Diabetes & 10 & $(13 \%)$ & \\
\hline Hypercholesterolemia & 5 & $(7 \%)$ & \\
\hline Hypertriglyceridemia & 18 & $(24 \%)$ & \\
\hline Vitamin D deficiency & 40 & $(53 \%)$ & \\
\hline Anemia & 51 & $(67 \%)$ & \\
\hline Hypertension (awake) & 23 & $(32 \%)$ & \\
\hline Hypertension (asleep) & 41 & $(59 \%)$ & \\
\hline \multicolumn{4}{|l|}{ Diagnosis } \\
\hline Mild cognitive impairment & 33 & $(43 \%)$ & \\
\hline Vascular dementia & 9 & $(12 \%)$ & \\
\hline Alzheimer's disease & 7 & $(9 \%)$ & \\
\hline Dementia with Lewy bodies & 1 & $(1 \%)$ & \\
\hline Mixed dementia & 26 & (34\%) & \\
\hline
\end{tabular}

*Montgomery-Asberg Depression Rating Scale. IQR, Interquartile range.

TABLE 2 | Sleep Apnea Syndrome.

\begin{tabular}{lccc}
\hline & \multicolumn{3}{c}{ Cohort ( $\boldsymbol{n}=\mathbf{7 6})$} \\
\cline { 2 - 4 } & Median & [IQR] & (Range) \\
\hline Blood oxygen saturation $\left(\mathrm{SpO}_{2}, \%\right)$ & 95 & {$[93-96]$} & $(87-98)$ \\
Sleep time with hypoxia*(\%) & 2 & {$[1-7]$} & $(0-54)$ \\
Number of events per night & 56 & {$[22-113]$} & $(2-548)$ \\
Apnea-hypopnea index (AHI) & 16 & {$[5-35]$} & $(1-73)$ \\
& $\boldsymbol{n}$ & $\mathbf{( \% )}$ & \\
SAS severity (AHI) & & & \\
None (<5) & 19 & $(25 \%)$ & \\
Mild (5-15) & 18 & $(24 \%)$ & \\
Moderate (15-30) & 7 & $(9 \%)$ & \\
Severe (>30) & 32 & $(42 \%)$ & \\
Sleep Apnea Syndrome (SAS) & 48 & $(63 \%)$ & \\
Obstructive & 31 & $(41 \%)$ \\
Central & 17 & $(22 \%)$ & \\
\hline
\end{tabular}

*Hypoxia defined as a reduction of SpO2 by 10\%. IQR, Interquartile range. written in different (wrong) colors and repress the wrong answer (Stroop interference effect). For all tests, the delay before the answer, and the number of uncorrected mistakes, are reported. (Al Lawati et al., 2009) Montgomery-Asberg Depression rating scale [MADRS (Montgomery and Asberg, 1979)], on 60 points, and Goldberg anxiety scale (Huber et al., 1999) on nine points, to rule out effects of depression or anxiety on cognitive test results.

Final etiological diagnosis of cognitive impairment was based on the neurocognitive assessments, as well as MRI (Siemens Avanto 27780, Munich, Germany) results and history of geriatric consultations. Mild cognitive impairment was diagnosed following the criteria of Petersen et al. (2001), while Alzheimer's disease $(\mathrm{AD})$ and $\mathrm{AD}$-like diseases were diagnosed according to the Diagnostic and Statistical Manual of Mental Disorders, 4th Edition (DSM-IV) and the recommendations of the National Institute on Aging - Alzheimer's Association workgroups (McKhann et al., 2011).

Vascular risk factors for the development of AD (Yaffe et al., 2011) were diagnosed based on a comprehensive assessment performed in our geriatric unit. Blood samples were obtained after a minimum of $10 \mathrm{~h}$ of fasting. The diagnosis of vitamin $\mathrm{D}\left[25(\mathrm{OH}) \mathrm{D}_{3}\right]$ deficiency was given if blood measurements were below $30 \mathrm{ng} / \mathrm{mL}$, diabetes was diagnosed with blood glucose levels above $7 \mathrm{mmol} / \mathrm{L}$ and anemia with hemoglobin levels below 12 (women) or 13 (men) g/dL. Likewise, the reference range was $4.1-6.5 \mathrm{mmol} / \mathrm{L}$ for total cholesterol and $0.6-1.8 \mathrm{mmol} / \mathrm{L}$ for triglycerides. Inflammation was diagnosed if the blood levels of C-reactive protein (CRP) was higher than $10 \mathrm{mg} / \mathrm{L}$ (McMorris et al., 2017) as per the recommendations of the World Health Organization (World Health Organization [WHO], 2014), and leucocyte concentration was higher than $100000 / \mathrm{mm}^{3}$. The blood pressure of patients was measured during waking hours, and after polygraphy, during night-time hours.

Written informed consent was obtained from all patients for their participation and confirmed by their next-of-kin if necessary. The study protocol was approved by an independent Ethical Review Board (CPP Amiens: 2015/6) and the national Data Protection Authority (CNIL:150075B-31). The study was registered in clinicaltrials.gov (NCT02578303). All

TABLE 3 | Cognitive Tests.

\begin{tabular}{lccc}
\hline & \multicolumn{3}{c}{ Cohort $(\boldsymbol{n}=\mathbf{7 6})$} \\
\cline { 2 - 4 } & Median & [IQR] & (Range) \\
\hline Mini-mental state exam (MMSE/30) & 23 & {$[19-26]$} & $(7-30)$ \\
Mattis dementia rating scale (MDRS/144) & 121 & {$[109-132]$} & $(60-143)$ \\
Stroop color and word test & & & \\
Colors (delay, s) & 19 & {$[15-29]$} & $(6-70)$ \\
Colors (non-corrected mistakes) & 0 & {$[0-0]$} & $(0-6)$ \\
Words (delay, s) & 34 & {$[25-50]$} & $(14-147)$ \\
Words (non-corrected mistakes) & 0 & {$[0-1]$} & $(0-9)$ \\
Interference (delay, s) & 53 & {$[38-76]$} & $(15-116)$ \\
Interference (non-corrected mistakes) & 4 & {$[1-13]$} & $(0-24)$ \\
\hline
\end{tabular}

$I Q R$, Interquartile range. 
procedures were performed in accordance with the 1964 Helsinki declaration.

\section{Statistical Analysis}

Descriptive statistics were used to summarize the data. ShapiroWilk tests were used to assess the normality of distributions. Due to skewed distribution of continuous variables, the data was presented as median [IQR]. Univariable regressions were performed to determine associations between five outcomes (MMSE, MDRS and Stroop Colors, Words, and Interference tests) and seventeen independent variables (age, BMI, sex, diagnosis of SAS, OSAS, or CSAS, AHI, sleep apnea severity, blood oxygen saturation, sleep time with hypoxia, number of events per night, diabetes, inflammation, hypercholesterolemia, hypertriglyceridemia, vitamin D deficiency, and anemia). Variables that do not measure sleep apnea were included because they represent cardiovascular risk factors known to affect sleep apnea or cognition in elderly patients (Fusetti et al., 2012). Multivariable regressions were then performed after backward selection (criterion $p<0.15$ ). Hypercholesterolemia was excluded from the multivariable models due to a low incidence $(n<10)$ in our cohort. Sample size of 47 patients was calculated to be sufficient to show even the moderate negative correlation between the MDRS and the AHI with $80 \%$ power and $5 \%$ significance. Therefore, our sample size of 76 was deemed sufficient for analysis, considering a probably high failure rate in the neurocognitive tests due to the age of the patients. Statistical analyses were performed using $\mathrm{R}$ version 3.3.2 (R Foundation for
Statistical Computing, Vienna, Austria). $P$-values $<0.05$ were considered statistically significant.

\section{RESULTS}

The final cohort comprised 58 women (76\%) and 18 men (24\%), aged 84 [IQR, 80-88] years (range, 73-96), with BMI 24 [IQR, 21-27] $\mathrm{kg} / \mathrm{m}^{2}$ (range, 15.6-40) (Table 1). Overall, they were not depressed (MADRS:6, IQR, 3-11; range, 0-31) nor anxious (Goldberg:3, IQR, 1-5; range, 0-9). A number of patients presented comorbidities, including heart failure $(11.8 \%)$, aortic stenosis $(3.9 \%)$, mitral stenosis $(1.3 \%)$, carotid stenosis $(3.9 \%)$, cerebral hematoma (3.9\%), transient ischemic accident $(3.9 \%)$, and cancer $(25 \%)$. The prevalence of arterial hypertension was $32 \%$ while awake but rose to $59 \%$ while asleep. Thirty-three patients (43\%) had mild cognitive impairment, 9 (12\%) had vascular dementia, 7 (9\%) had Alzheimer's disease, 1 (1\%) had Lewy-body dementia, and 26 (34\%) had mixed dementia.

The median AHI was 16.4 (IQR, 5.4-35.3; range, 0.5-73), and indicated severe sleep apnea in 32 patients (42\%) (Table 2). SAS was diagnosed in 48 patients (63\%), of which 31 (41\%) with OSAS and $17(22 \%)$ with CSAS.

The median MMSE score was 22.5 (IQR, 19-26; range, 730 ), with 41 (54\%) patients scoring $\leq 23$ (Table 3). Univariable regression revealed the MMSE score to be associated with sex and diabetes (Table 4). There was no significant difference in MMSE between patients with moderate to severe SAS and patients with

TABLE 4 | Uni- and Multi-variable regression to identify factors associated with the Mini Mental State Exam (MMSE).

\begin{tabular}{|c|c|c|c|c|c|c|}
\hline & \multicolumn{3}{|c|}{ Univariable } & \multicolumn{3}{|c|}{ Multivariable $(n=71)$ Backward selection } \\
\hline & Regression coefficient & 95\% C.I. (range) & $p$-Value & Regression coefficient & 95\% C.I. (range) & $p$-Value \\
\hline Age & -0.19 & $(-0.39$ to 0.02$)$ & 0.072 & -0.25 & $(-0.45$ to -0.05$)$ & 0.014 \\
\hline $\mathrm{BMI}\left(\mathrm{kg} / \mathrm{m}^{2}\right)$ & 0.14 & $(-0.09$ to 0.37$)$ & 0.226 & & & \\
\hline Female Gender & -2.72 & $(-5.37$ to 0.08$)$ & 0.044 & & & \\
\hline SAS & 0.07 & $(-2.22$ to 2.35$)$ & 0.955 & & & \\
\hline OSAS & -0.29 & $(-2.58$ to 2.00$)$ & 0.801 & & & \\
\hline CSAS & 0.92 & $(-1.86$ to 3.70$)$ & 0.510 & & & \\
\hline Apnea-hypopnea index (AHI) & 0.01 & $(-0.05$ to 0.07$)$ & 0.723 & 0.04 & $(-0.01-0.10)$ & 0.142 \\
\hline \multicolumn{7}{|l|}{ Sleep apnea severity } \\
\hline None & REF & & & & & \\
\hline Mild & 1.11 & $(-2.08$ to 4.31$)$ & 0.489 & & & \\
\hline Moderate & -3.44 & $(-7.90$ to 1.01$)$ & 0.128 & & & \\
\hline Severe & 0.35 & $(-2.46$ to 3.15$)$ & 0.806 & & & \\
\hline Blood oxygen saturation (SpO2,\%) & -0.07 & $(-0.60$ to 0.45$)$ & 0.787 & & & \\
\hline Sleep time with hypoxia* (\%) & 0.01 & $(-0.07$ to 0.10$)$ & 0.738 & & & \\
\hline Number of events per night & 0.00 & $(-0.01$ to 0.01$)$ & 0.438 & & & \\
\hline Diabetes & -4.02 & $(-7.30$ to -0.73$)$ & 0.017 & -4.91 & $(-8.10$ to -1.72$)$ & 0.003 \\
\hline Inflammation & 1.96 & $(-0.33$ to 4.25$)$ & 0.092 & & & \\
\hline Hypercholesterolemia** & -0.83 & $(-5.31$ to 3.65$)$ & 0.712 & & & \\
\hline Hypertriglyceridemia & -0.89 & $(-3.58$ to 1.79$)$ & 0.508 & & & \\
\hline Vitamin D deficiency & 1.17 & $(-1.09$ to 3.44$)$ & 0.304 & 1.83 & $(-0.33$ to 4.00$)$ & 0.096 \\
\hline Anemia & 0.17 & $(-2.26$ to 2.59$)$ & 0.892 & & & \\
\hline
\end{tabular}

*Hypoxia defined as a reduction of SpO2 by $10 \%$. ** Excluded from multivariable analysis due to low incidence $(<10)$. 
mild to no SAS (Figure 2). Multivariable regression analysis revealed that MMSE decreased with age $(\beta=-0.25, \mathrm{CI},-0.45$ to $-0.05 ; p=0.014)$ and in patients with diabetes $(\beta=-4.91, C I$, -8.10 to $-1.72 ; p=0.01$.

The median MDRS score was 120.5 (IQR, 109.3-132.0; range, 60-143) (Table 3). Univariable regression revealed the MDRS to be associated with SAS, OSAS, AHI, diabetes, and hypertriglyceridemia (Table 5). The MDRS score was lower for patients with moderate to severe SAS compared to patients with mild to no SAS (Figure 2; $p=0.009$ ). Multivariable regression analysis revealed that MDRS decreased with age $(\beta=-0.81, \mathrm{CI}$, -1.54 to $-0.08 ; p=0.031)$, in patients with OSAS $(\beta=-10.03$, $\mathrm{CI},-18.26$ to $-1.80 ; p=0.018)$, and in patients with diabetes $(\beta=-16.10, \mathrm{CI},-27.8$ to $-4.36 ; p=0.008)$.

Twenty patients were unable to perform the Stroop test. For the remaining patients, the median Stroop Colors delay was $19 \mathrm{~s}$ (IQR, 15-29; range, 6-70) and the median number of mistakes was 0 (IQR, 0-0; range, 0-6) (Table 3). The Stroop Words delay was $34 \mathrm{~s}$ (IQR, 25-50; range, 14-147) and the median number of mistakes was 0 (IQR, 0-1; range, 0-9). The Stroop Interference delay was 53 s (IQR, 38-76; range, 15-116) and the median number of mistakes was 4 (IQR, 1-13; range, $0-24)$. Univariable regressions revealed the Stroop Colors delay to be associated with age, AHI, severe SAS, and anemia (Table 6), the Stroop

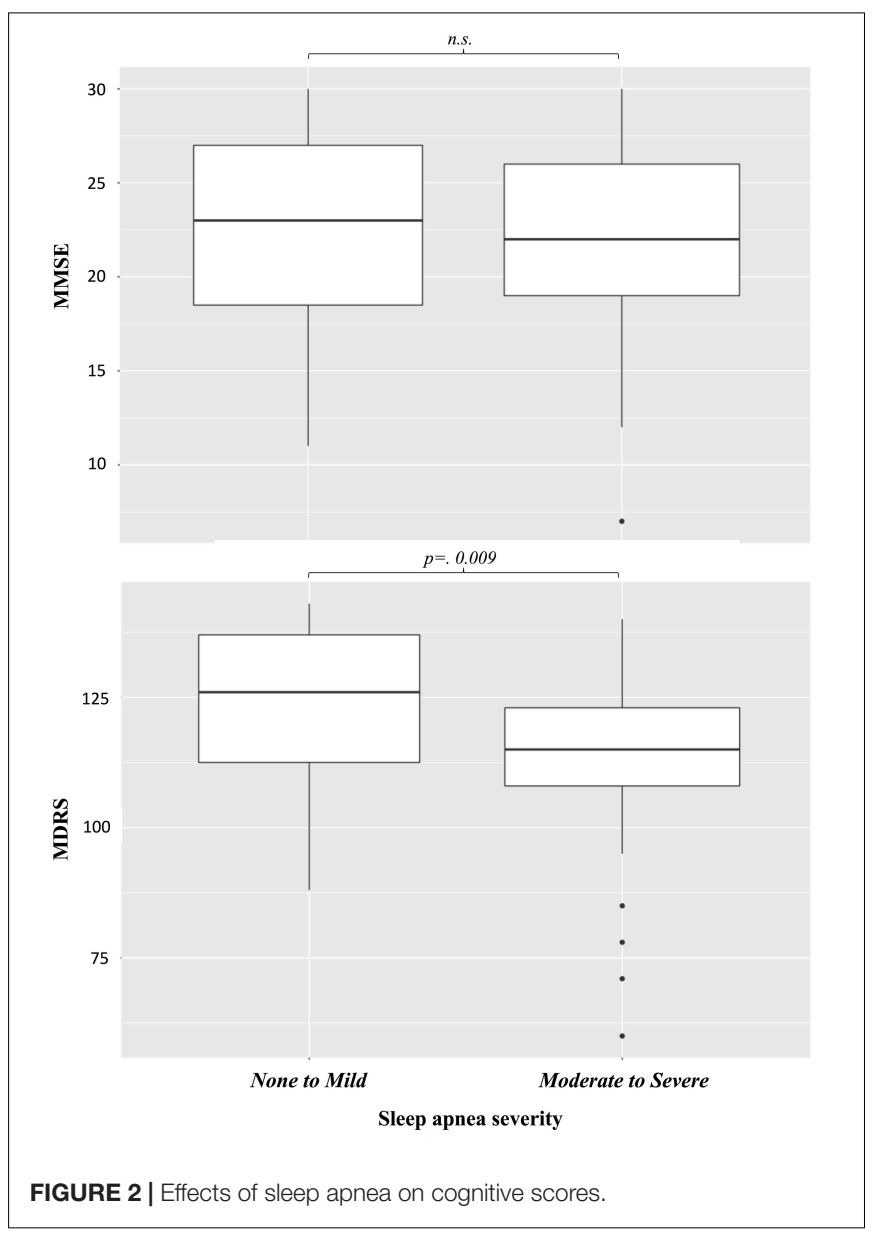

Words delay to be associated with AHI (Table 7), and the Stroop Interference delay to be associated with SAS, CSAS, AHI and diabetes (Table 8). All three delays were increased in patients with moderate to severe SAS compared to patients with mild to no SAS (Figure 3, Color, $p=0.022$, Word, $p=0.031$, Interference, $p=0.031)$. Multivariable regressions revealed that Stroop Color delay increased with age $(\beta=0.64, \mathrm{CI}, 0.09-1.19 ; p=0.024)$ and AHI $(\beta=0.17, \mathrm{CI}, 0.02-0.32 ; p=0.030)$, that Stroop Words delay increased with AHI $(\beta=0.31, \mathrm{CI}, 0.00-0.62 ; p=0.047)$ and that Stroop Interference delay increased in patients with CSAS $(\beta=24.45, C I, 15.21-50.55 ; p=0.0024)$ and in patients with diabetes $(\beta=32.88, C I, 15.21-50.55 ; p=0.001)$.

\section{DISCUSSION}

The prevalence of SAS in elderly patients admitted to our geriatric unit was 63\% (OSAS in $41 \%$ and CSAS in 22\%), which is considerably higher than that reported in the general population, between 24 and 40\% (Peppard et al., 2013; Heinzer et al., 2015). Our results demonstrated the preeminence of SAS among factors associated with cognitive deficit thus confirming previous observations from the literature (Olaithe and Bucks, 2013; Aoki et al., 2014; Arli et al., 2015; Osorio et al., 2015). It is worth noting that the prevalence of SAS in our cohort remains higher than recent estimates for the elderly population (25\% for men and $42 \%$ for women) (Heinzer et al., 2015), which could be due to our great proportion of patients with cognitive deficit (Moran et al., 2005; Emamian et al., 2016). Interestingly, this finding is in agreement with recent observations from German geriatric wards (Gronewold et al., 2019). This study is nevertheless the first to investigate comorbidities associating with SAS while differentiating OSAS and CSAS in this particular population.

The patients in this cohort presented various levels of cognitive deficit, although all were diagnosed with disorders ranging from MCI to AD or vascular dementia. Interestingly, in this diverse cohort, OSAS was identified as an independent predictor of poor MDRS scores but was not associated with MMSE scores. While both tests are used to detect cognitive impairment in the elderly, there are indications that they may not evaluate the same cognitive domains (Freidl et al., 1996), or that the MDRS may be more specific and sensitive (Kaszas et al., 2012; Holden et al., 2016). Furthermore, the ability of the MMSE to efficiently screen for cognitive impairment in the elderly has recently been put into question (Gagnon et al., 2018). It is important to note that the fact that CSAS did not affect MDRS scores in our study could be due to its lower incidence in our cohort and is no proof of lack of effect. Our results also revealed that $\mathrm{AHI}$ associates with impaired attention, indicated by the increased delay in the Stroop Colors and Words tests, and that CSAS and perhaps OSAS associate with impaired executive function, indicated by the increased delay in the Stroop Interference test. This is in line with the literature reporting that SAS affects vigilance and the attentional domain (Beebe et al., 2003) and executive function (Beebe et al., 2003; Olaithe and Bucks, 2013). In 2012, Bucks et al. (2013) suggested that OSAS may affect vigilance through sleep fragmentation 
TABLE 5 | Uni- and Multi-variable regression to identify factors associated with the Mattis Dementia Rating Scale (MDRS).

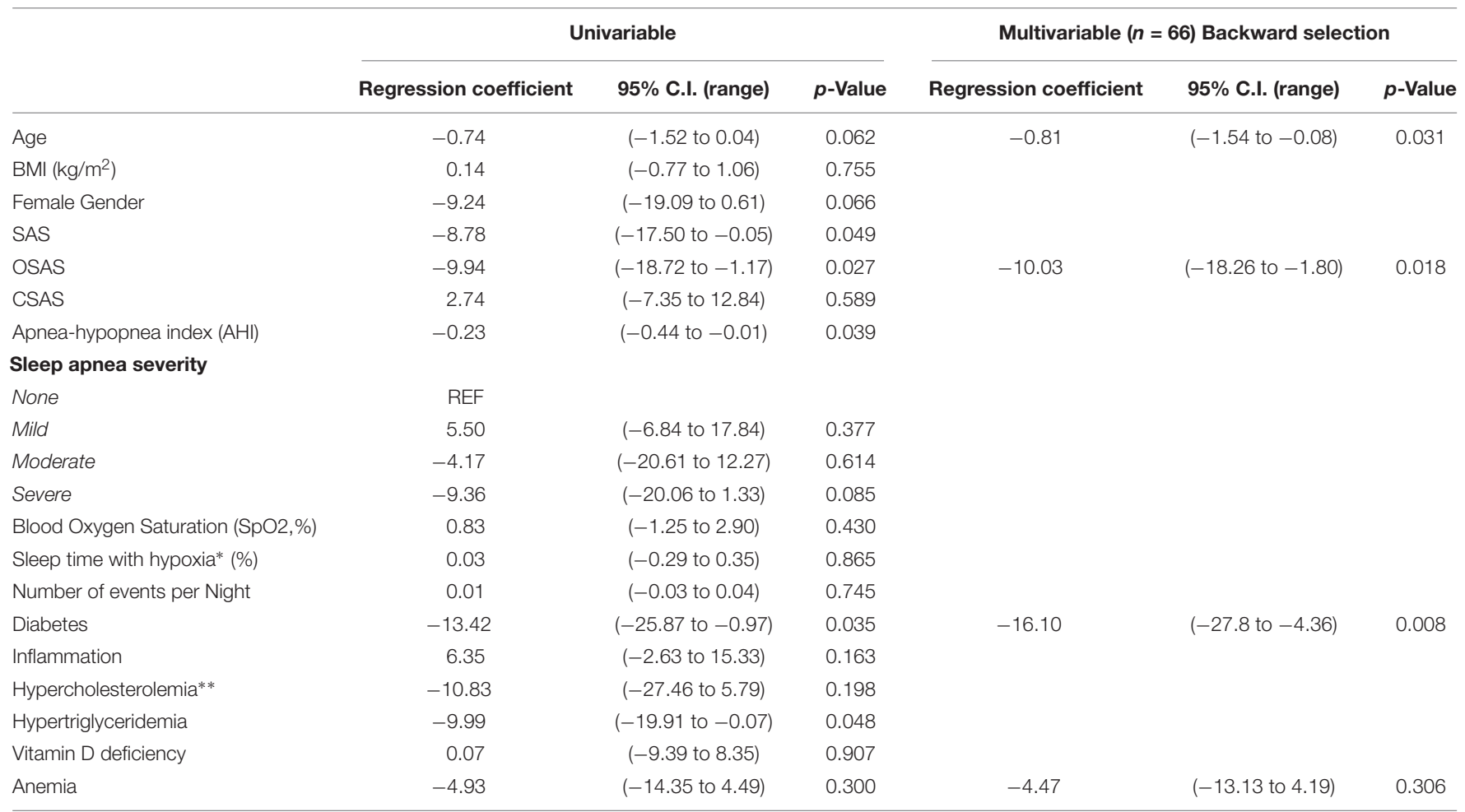

*Hypoxia defined as a reduction of SpO2 by $10 \% .{ }^{* *}$ Excluded from multivariable analysis due to insufficient number of observations $(<10)$.

TABLE 6 | Uni- and Multi-variable regression to identify factors associated with the Stroop Colors Test (delay, s).

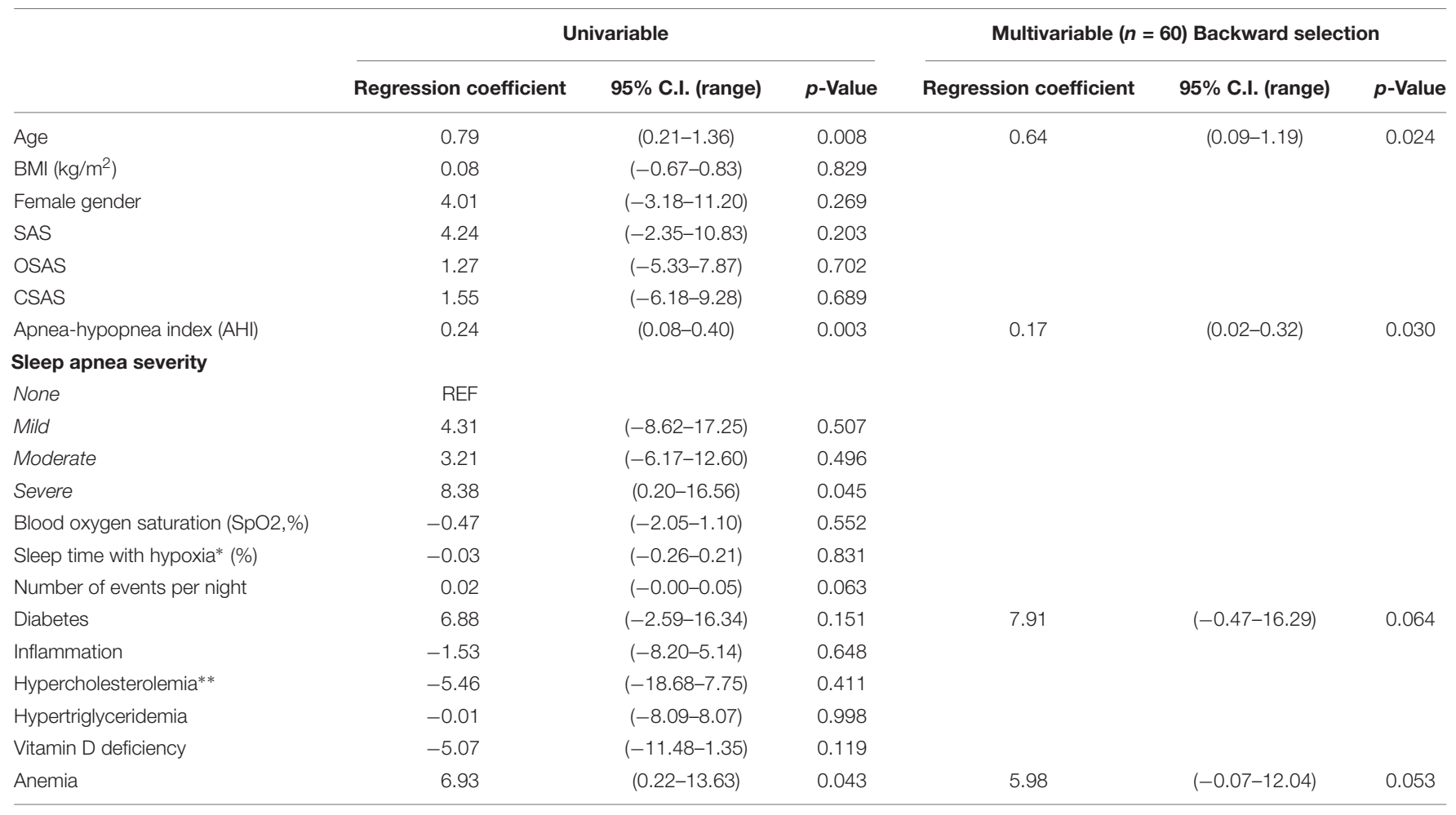

*Hypoxia defined as a reduction of SpO2 by 10\%. ** Excluded from multivariable analysis due to low incidence $(<10)$. 
TABLE 7 | Uni- and Multi-variable regression to identify factors associated with the Stroop Words Test (delay, s).

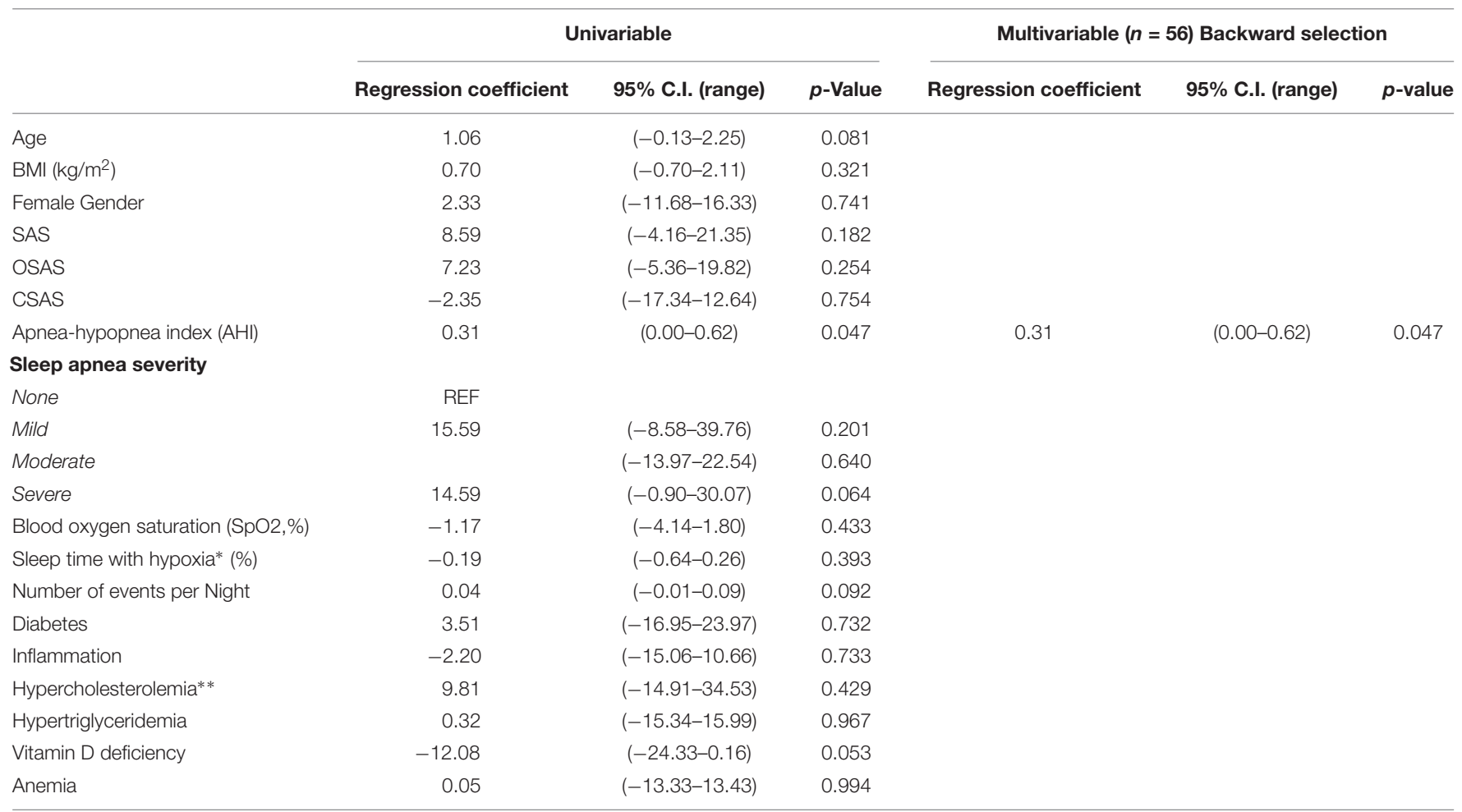

*Hypoxia defined as a reduction of SpO2 by $10 \%$. ** Excluded from multivariable analysis due to low incidence $(<10)$.

TABLE 8 | Uni- and Multi-variable regression to identify factors associated with the Stroop Interference Test (delay, s).

\begin{tabular}{|c|c|c|c|c|c|c|}
\hline & \multicolumn{3}{|c|}{ Univariable } & \multicolumn{3}{|c|}{ Multivariable $(n=50)$ Backward selection } \\
\hline & Regression coefficient & 95\% C.I. (range) & $p$-Value & Regression coefficient & 95\% C.I. (range) & $p$-value \\
\hline Age & 1.29 & $(-0.05-2.63)$ & 0.059 & 1.13 & $(-0.05-2.30)$ & 0.061 \\
\hline $\mathrm{BMI}\left(\mathrm{kg} / \mathrm{m}^{2}\right)$ & 0.12 & $(-1.41-1.66)$ & 0.872 & & & \\
\hline Female Gender & 5.74 & $(-9.76-21.24)$ & 0.460 & & & \\
\hline SAS & 16.93 & (3.21-30.66) & 0.017 & & & \\
\hline OSAS & 1.98 & $(-12.05-16.01)$ & 0.778 & 13.57 & $(-0.14-27.28)$ & 0.052 \\
\hline CSAS & 17.18 & $(2.02-32.33)$ & 0.027 & 24.45 & $(8.42-40.49)$ & 0.004 \\
\hline Apnea-hypopnea index (AHI) & 0.45 & $(-0.11-0.78)$ & 0.011 & & & \\
\hline \multicolumn{7}{|l|}{ Sleep apnea severity } \\
\hline None & REF & & & & & \\
\hline Mild & -6.02 & $(-26.26-14.23)$ & 0.553 & & & \\
\hline Moderate & 7.65 & $(-20.32-35.62)$ & 0.585 & & & \\
\hline Severe & 14.15 & $(-3.65-31.95)$ & 0.116 & & & \\
\hline Blood Oxygen Saturation (SpO2,\%) & -0.33 & $(-3.58-2.93)$ & 0.840 & & & \\
\hline Sleep Time with hypoxia* (\%) & 0.03 & $(-0.44-0.51)$ & 0.890 & & & \\
\hline Number of events per Night & 0.04 & $(-0.02-0.09)$ & 0.189 & & & \\
\hline Diabetes & 28.53 & $(-8.65-48.41)$ & 0.006 & 32.88 & $(15.21-50.55)$ & 0.001 \\
\hline Inflammation & -0.52 & $(-14.80-13.77)$ & 0.942 & & & \\
\hline Hypercholesterolemia** & -12.64 & $(-42.31-17.02)$ & 0.395 & & & \\
\hline Hypertriglyceridemia & 13.97 & $(-2.22-30.16)$ & 0.089 & & & \\
\hline Vitamin D deficiency & -6.85 & $(-20.71-7.02)$ & 0.326 & & & \\
\hline Anemia & 8.65 & $(-5.91-23.21)$ & 0.238 & & & \\
\hline
\end{tabular}

*Hypoxia defined as a reduction of SpO2 by $10 \%$.**Excluded from multivariable analysis due to low incidence (<10). 


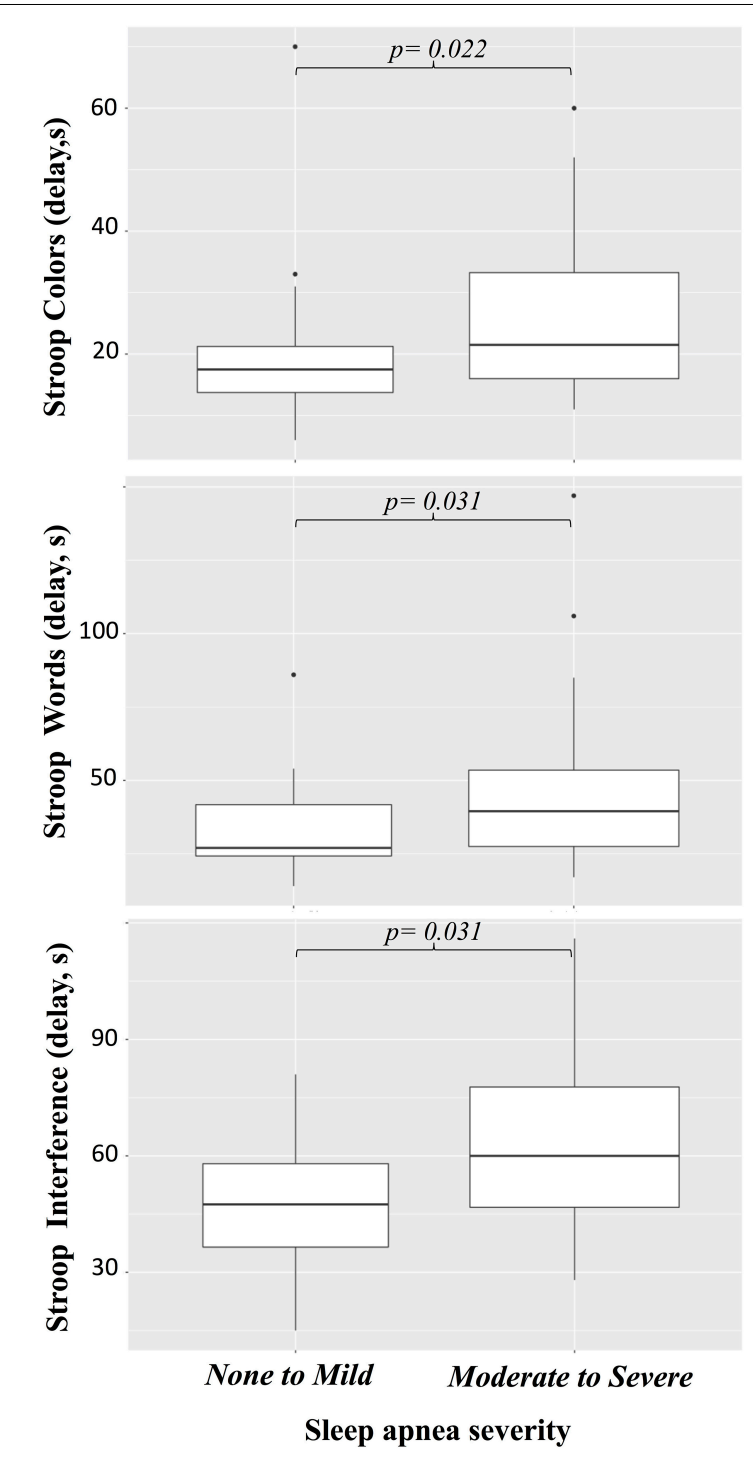

FIGURE 3 | Effects of sleep apnea on Stroop tests.

and executive function through hypoxia. In this study, we measured hypoxia and sleep apnea severity (AHI) separately but were not able to verify this hypothesis. It is unclear why, unlike previous literature, AHI rather than oxygen desaturation was associated with cognitive deficit in our cohort, though it could be due to the different manifestations in SAS in the elderly population and in the general population (Al Lawati et al., 2009; Heinzer et al., 2015). The relationship between intermittent hypoxia and cognition remain complex, however, and animal studies have suggested that intermittent hypoxia may not directly impair brain structure (Jorba et al., 2017), but may play a role in the etiology of Alzheimer's Disease (Shiota et al., 2013; Menal et al., 2018; Macheda et al., 2019). Nevertheless, fragmented sleep and excessive daytime sleepiness have been shown to modify the relationship between AHI and several co-morbidities (Nena et al., 2012; Ren et al., 2016) and could contribute to cognitive decline in SAS patients (Zhou et al., 2016). Unfortunately, daytime sleepiness was not evaluated in this study, and could therefore not be included in the multivariable model. We found no association between the number of events per night and cognitive outcomes, though this variable may have underestimated sleep fragmentation, as it did not take snoring into account. The interaction between AHI, sleep fragmentation, daytime sleepiness, and cognitive decline in the elderly remains therefore a question for further search.

We decided to use vascular risk factors known to associate with SAS or AD to adjust the regression models for our cognitive tests. Interestingly, only diabetes independently associated with reduced MMSE and MDRS scores, as well as increased Stroop delay. Taken together, OSAS and diabetes are associated with a reduction of 26 points in the MDRS. Interestingly, severe sleep apnea was shown to be associated with diabetes in the general population (Heinzer et al., 2015). This preeminence of diabetes among risk factors for cognitive deficit has been recently demonstrated (Musen et al., 2018) and our results indicate that sleep apnea should be investigated among the other risk factors for the cognitive deficit of the elderly. Indeed, sleep apnea treatment with continuous positive air pressure has shown success in slowing down cognitive decline in the elderly (Kushida et al., 2012; Troussiere et al., 2014; Osorio et al., 2015), so that sleep apnea could be considered a modifiable risk factor for cognitive decline in the elderly (Ancoli-Israel et al., 2008).

This study is among the first to combine a comprehensive measure of sleep apnea, including the AHI, sleep hypoxia and events per night, with a multivariable regression analysis including known vascular risk factors to determine associations with cognitive deficit in cohort of elderly patients suffering from diffuse cognitive impairment. Moreover, the cohort was controlled for depression and anxiety. The fact that sleep apnea was selected among other well-established risk factors is a powerful demonstration of its association with cognitive deficit in these patients and suggests that sleep apnea ought to be screened for and treated in priority when possible. Further, our distinction of patients suffering primarily from OSAS or CSAS offers novel insight into the effects of these different, yet oftentimes overlapping disorders.

However, this study has several limitations. First, the age of the patients and their cognitive impairment caused a large number of failures of the neurocognitive assessment, especially for the Stroop test. This has weakened our regression analyses and increased the chance of underestimating associations. Second, the fact that patients unable to complete the neurocognitive tests were excluded from the study has created a potential bias, since these patients were likely to exhibit more severe cognitive symptoms. Third, no additional measure of dementia was used to validate MDRS results and assess dementia severity. Fourth, the prevalence of women in our cohort represents the population admitted to our institution, and our results may not be applicable for younger sleep apnea patients, which are predominantly men. Finally, our list of competing risk factors was incomplete, as we were unable to correctly or systematically assess the patients' smoking habits, alcohol consumption, physical inactivity, or daytime sleepiness. 


\section{CONCLUSION}

This study reveals the high prevalence of sleep apnea in elderly patients with cognitive impairment, and demonstrates the independent association between sleep apnea and cognitive deficit. OSAS was associated with a reduction of the MDRS score, while CSAS was associated with an increased Stroop Interference delay. Our data suggest that elderly patients with cognitive deficit should be screened for sleep apnea and encouraged to follow treatments, if necessary, to prevent cognitive decline.

\section{DATA AVAILABILITY STATEMENT}

The datasets generated for this study are available on request to the corresponding author.

\section{ETHICS STATEMENT}

This work was completed after being approved by an independent Ethical Review Board (CPP Amiens: 2015/6) and the national Data Protection Authority (CNIL:150075B-31). The study was

\section{REFERENCES}

Al Lawati, N. M., Patel, S. R., and Ayas, N. T. (2009). Epidemiology, risk factors, and consequences of obstructive sleep apnea and short sleep duration. Prog. Cardiovasc. Dis. 51, 285-293. doi: 10.1016/j.pcad.2008. 08.001

Ancoli-Israel, S., Palmer, B. W., Cooke, J. R., Corey-Bloom, J., Fiorentino, L., Natarajan, L., et al. (2008). Cognitive effects of treating obstructive sleep apnea in Alzheimer's disease: a randomized controlled study. J. Am. Geriatr. Soc. 56, 2076-2081. doi: 10.1111/j.1532-5415.2008.01934.x

Aoki, K., Matsuo, M., Takahashi, M., Murakami, J., Aoki, Y., Aoki, N., et al. (2014). Association of sleep-disordered breathing with decreased cognitive function among patients with dementia. J. Sleep Res. 23, 517-523. doi: 10.1111/jsr.12167

Arli, B., Bilen, S., Titiz, A. P., Ulusoy, E. K., Mungan, S., Gurkas, E., et al. (2015). Comparison of cognitive functions between obstructive sleep apnea syndrome and simple snoring patients: OSAS may be a modifiable risk factor for cognitive decline. Appl. Neuropsychol. Adult 22, 282-286. doi: 10.1080/23279095.2014. 925901

Beebe, D. W., Groesz, L., Wells, C., Nichols, A., and McGee, K. (2003). The neuropsychological effects of obstructive sleep apnea: a meta-analysis of normreferenced and case-controlled data. Sleep 26, 298-307. doi: 10.1093/sleep/26. 3.298

Bubu, O. M., Pirraglia, E., Andrade, A. G., Sharma, R. A., Gimenez-Badia, S., Umasabor-Bubu, O. Q., et al. (2019). Obstructive sleep apnea and longitudinal Alzheimer's disease biomarker changes. Sleep 42:zsz048. doi: 10.1093/sleep/ zsz048

Bucks, R. S., Olaithe, M., and Eastwood, P. (2013). Neurocognitive function in obstructive sleep apnoea: a meta-review. Respirology 18, 61-70. doi: 10.1111/ j.1440-1843.2012.02255.x

Comalli, P. E. Jr., Wapner, S., and Werner, H. (1962). Interference effects of Stroop color-word test in childhood, adulthood, and aging. J. Genet. Psychol. 100, 47-53. doi: 10.1080/00221325.1962.10533572

Cooke, J. R., Ayalon, L., Palmer, B. W., Loredo, J. S., Corey-Bloom, J., Natarajan, L., et al. (2009). Sustained use of CPAP slows deterioration of cognition, sleep, and mood in patients with Alzheimer's disease and obstructive sleep apnea: a preliminary study. J. Clin. Sleep Med. 5, 305-309.

Dostalova, V., Koleckarova, S., Kuska, M., Pretl, M., and Bezdicek, O. (2019). Effects of continuous positive airway pressure on neurocognitive and neuropsychiatric function in obstructive sleep apnea. J. Sleep Res. 28:e12761. doi: $10.1111 /$ jsr. 12761 registered in clinicaltrials.gov (NCT02578303) and has not been submitted to any other journal.

\section{AUTHOR CONTRIBUTIONS}

JA-Z designed the study, collected the data, and edited the manuscript. J-MS designed the study and edited the manuscript. JV contributed to statistical analyses, interpretation of data, and manuscript writing. MS interpreted the data, wrote and edited the manuscript. YD collected the data and edited the manuscript. KM contributed to statistical analyses and manuscript editing. OB designed the study, interpreted the data, and wrote and edited the manuscript. All authors approved and accountability for final version of the manuscript.

\section{ACKNOWLEDGMENTS}

The authors would like to thank Ms. Ingrid Gyselinck, Mr. Momar Diouf, and Mr. Salif Dao for their contribution with data collection.

Emamian, F., Khazaie, H., Tahmasian, M., Leschziner, G. D., Morrell, M. J., Hsiung, G. Y., et al. (2016). The association between obstructive sleep apnea and Alzheimer's disease: a meta-analysis perspective. Front. Aging Neurosci. 8:78. doi: 10.3389/fnagi.2016.00078

Freidl, W., Schmidt, R., Stronegger, W. J., Fazekas, F., and Reinhart, B. (1996). Sociodemographic predictors and concurrent validity of the mini mental state examination and the mattis dementia rating scale. Eur. Arch. Psychiatry Clin. Neurosci. 246, 317-319. doi: 10.1007/bf02189025

Fusetti, M., Fioretti, A. B., Valenti, M., Masedu, F., Lauriello, M., and Pagliarella, M. (2012). Cardiovascular and metabolic comorbidities in patients with obstructive sleep apnoea syndrome. Acta Otorhinolaryngol. Ital. 32, 320-325.

Gagnon, K., Baril, A. A., Montplaisir, J., Carrier, J., Chami, S., Gauthier, S., et al. (2018). Detection of mild cognitive impairment in middle-aged and older adults with obstructive sleep apnoea. Eur. Respir. J. 52:1801137. doi: 10.1183/ 13993003.01137-2018

Gardner, R. Jr., Oliver-Munoz, S., Fisher, L., and Empting, L. (1981). Mattis dementia rating scale: internal reliability study using a diffusely impaired population. J. Clin. Neuropsychol. 3, 271-275. doi: 10.1080/01688638108403130

Gronewold, J., Haensel, R., Kleinschnitz, C., Frohnhofen, H., and Hermann, D. M. (2019). Sleep-disordered breathing in hospitalized geriatric patients with mild dementia and its association with cognition, emotion and mobility. Int. J. Environ. Res. Public Health 16:863. doi: 10.3390/ijerph16050863

Heinzer, R., Vat, S., Marques-Vidal, P., Marti-Soler, H., Andries, D., Tobback, N., et al. (2015). Prevalence of sleep-disordered breathing in the general population: the HypnoLaus study. Lancet Respir. Med. 3, 310-318. doi: 10.1016/s22132600(15)00043-0

Hersch, E. L. (1979). Development and application of the extended scale for dementia. J. Am. Geriatr. Soc. 27, 348-354. doi: 10.1111/j.1532-5415.1979. tb06056.x

Holden, S. K., Jones, W. E., Baker, K. A., Boersma, I. M., and Kluger, B. M. (2016) Outcome measures for Parkinson's disease dementia: a systematic review. Mov. Dis. Clin. Pract. 3, 9-18. doi: 10.1002/mdc3.12225

Huber, P., Mulligan, R., Mackinnon, A., Nebuloni-French, T., and Michel, J. P. (1999). Detecting anxiety and depression in hospitalised elderly patients using a brief inventory. Eur. Psychiatry J. Assoc. Eur. Psychiatr. 14, 11-16. doi: 10.1016/ s0924-9338(99)80710-x

Jorba, I., Menal, M. J., Torres, M., Gozal, D., Pinol-Ripoll, G., Colell, A., et al. (2017). Ageing and chronic intermittent hypoxia mimicking sleep apnea do not modify local brain tissue stiffness in healthy mice. J. Mech. Behav. Biomed. Mater. 71, 106-113. doi: 10.1016/j.jmbbm.2017.03.001 
Kalafat Michel, P. J., Hugonot-Diener, L., and Poitrenaud, J. (2003). The mini mental state (MMS): French standardization and normative data [Standardisation et étalonnage français du "Mini Mental State" (MMS) version GRÉCO]. Rev. Neuropsychol. 13, 209-236.

Kaszas, B., Kovacs, N., Balas, I., Kallai, J., Aschermann, Z., Kerekes, Z., et al. (2012). Sensitivity and specificity of Addenbrooke's cognitive examination, mattis dementia rating scale, frontal assessment battery and mini mental state examination for diagnosing dementia in Parkinson's disease. Parkinsonism Relat. Disord. 18, 553-556. doi: 10.1016/j.parkreldis.2012.02.010

Kushida, C. A., Nichols, D. A., Holmes, T. H., Quan, S. F., Walsh, J. K., Gottlieb, D. J., et al. (2012). Effects of continuous positive airway pressure on neurocognitive function in obstructive sleep apnea patients: the apnea positive pressure long-term efficacy study (APPLES). Sleep 35, 1593-1602.

Kuwabara, M., Tomitani, N., Shiga, T., and Kario, K. (2018). Polysomnographyderived sleep parameters as a determinant of nocturnal blood pressure profile in patients with obstructive sleep apnea. J. Clin. Hypertens. 20, 1039-1048. doi: $10.1111 /$ jch. 13308

Kylstra, W. A., Aaronson, J. A., Hofman, W. F., and Schmand, B. A. (2013). Neuropsychological functioning after CPAP treatment in obstructive sleep apnea: a meta-analysis. Sleep Med. Rev. 17, 341-347. doi: 10.1016/j.smrv.2012. 09.002

Liguori, C., and Placidi, F. (2018). Is it time to consider obstructive sleep apnea syndrome a risk factor for Alzheimer's disease? Am. J. Respir. Crit. Care Med. 197, 855-856. doi: 10.1164/rccm.201710-2105ed

Macheda, T., Roberts, K., Lyons, D. N., Higgins, E., Ritter, K. J., Lin, A. L., et al. (2019). Chronic intermittent hypoxia induces robust astrogliosis in an Alzheimer's disease-relevant mouse model. Neuroscience 398, 55-63. doi: 10. 1016/j.neuroscience.2018.11.040

Marshall, N. S., Barnes, M., Travier, N., Campbell, A. J., Pierce, R. J., McEvoy, R. D., et al. (2006). Continuous positive airway pressure reduces daytime sleepiness in mild to moderate obstructive sleep apnoea: a meta-analysis. Thorax 61, 430-434. doi: 10.1136/thx.2005.050583

Martin, M. S., Sforza, E., Roche, F., Barthelemy, J. C., and Thomas-Anterion, C. (2015). Sleep breathing disorders and cognitive function in the elderly: an 8year follow-up study. the proof-synapse cohort. Sleep 38, 179-187. doi: 10.5665/ sleep. 4392

McKhann, G. M., Knopman, D. S., Chertkow, H., Hyman, B. T., Jack, C. R. Jr., Kawas, C. H., et al. (2011). The diagnosis of dementia due to Alzheimer's disease: recommendations from the National Institute on Aging-Alzheimer's Association workgroups on diagnostic guidelines for Alzheimer's disease. Alzheimers Dement. J. Alzheimers Assoc. 7, 263-269. doi: 10.1016/j.jalz.2011.03.005

McMorris, T., Hale, B. J., Barwood, M., Costello, J., and Corbett, J. (2017). Effect of acute hypoxia on cognition: a systematic review and metaregression analysis. Neurosci. Biobehav. Rev. 74(Pt A), 225-232. doi: 10.1016/j.neubiorev.2017.01.019

Menal, M. J., Jorba, I., Torres, M., Montserrat, J. M., Gozal, D., Colell, A., et al. (2018). Alzheimer's disease mutant mice exhibit reduced brain tissue stiffness compared to wild-type mice in both normoxia and following intermittent hypoxia mimicking sleep apnea. Front. Neurol. 9:1. doi: 10.3389/fneur.2018. 00001

Montgomery, S. A., and Asberg, M. (1979). A new depression scale designed to be sensitive to change. Br. J. Psychiatry J. Ment. Sci. 134, 382-389. doi: 10.1192/ bjp.134.4.382

Moran, M., Lynch, C. A., Walsh, C., Coen, R., Coakley, D., and Lawlor, B. A. (2005). Sleep disturbance in mild to moderate Alzheimer's disease. Sleep Med. 6, 347-352. doi: 10.1016/j.sleep.2004.12.005

Musen, G., Tinsley, L. J., Marcinkowski, K. A., Pober, D., Sun, J. K., Khatri, M., et al. (2018). Cognitive function deficits associated with long-duration type 1 diabetes and vascular complications. Diabetes Care 41, 1749-1756. doi: 10.2337/dc171955

Nena, E., Steiropoulos, P., Papanas, N., Tsara, V., Fitili, C., Froudarakis, M. E., et al. (2012). Sleepiness as a marker of glucose deregulation in obstructive sleep apnea. Sleep Breath. 16, 181-186. doi: 10.1007/s11325-010-0472-y

Olaithe, M., and Bucks, R. S. (2013). Executive dysfunction in OSA before and after treatment: a meta-analysis. Sleep 36, 1297-1305. doi: 10.5665/sleep. 2950

Osorio, R. S., Gumb, T., Pirraglia, E., Varga, A. W., Lu, S. E., Lim, J., et al. (2015). Sleep-disordered breathing advances cognitive decline in the elderly. Neurology 84, 1964-1971. doi: 10.1212/WNL.0000000000001566
Peppard, P. E., Young, T., Barnet, J. H., Palta, M., Hagen, E. W., and Hla, K. M. (2013). Increased prevalence of sleep-disordered breathing in adults. Am. J. Epidemiol. 177, 1006-1014. doi: 10.1093/aje/kws342

Petersen, R. C., Doody, R., Kurz, A., Mohs, R. C., Morris, J. C., Rabins, P. V., et al. (2001). Current concepts in mild cognitive impairment. Arch. Neurol. 58, 1985-1992.

Ren, R., Li, Y., Zhang, J., Zhou, J., Sun, Y., Tan, L., et al. (2016). Obstructive sleep apnea with objective daytime sleepiness is associated with hypertension. Hypertension 68, 1264-1270. doi: 10.1161/hypertensionaha.115.06941

Richards, K. C., Gooneratne, N., Dicicco, B., Hanlon, A., Moelter, S., Onen, F., et al. (2019). CPAP adherence may slow 1-year cognitive decline in older adults with mild cognitive impairment and apnea. J. Am. Geriatr. Soc. 67, 558-564. doi: 10.1111 /jgs.15758

Rosenzweig, I., Glasser, M., Polsek, D., Leschziner, G. D., Williams, S. C., and Morrell, M. J. (2015). Sleep apnoea and the brain: a complex relationship. Lancet Respir. Med. 3, 404-414. doi: 10.1016/s2213-2600(15)00090-9

Shiota, S., Takekawa, H., Matsumoto, S. E., Takeda, K., Nurwidya, F., Yoshioka, Y., et al. (2013). Chronic intermittent hypoxia/reoxygenation facilitate amyloidbeta generation in mice. J. Alzheimers Dis. 37, 325-333. doi: 10.3233/JAD130419

Somers, V. K., White, D. P., Amin, R., Abraham, W. T., Costa, F., Culebras, A., et al. (2008). Sleep apnea and cardiovascular disease: an american heart association/American college of cardiology foundation scientific statement from the american heart association council for high blood pressure research professional education committee, council on clinical cardiology, stroke council, and council on cardiovascular nursing. in collaboration with the national heart, lung, and blood institute national center on sleep disorders research (national institutes of health). Circulation 118, 1080-1111. doi: 10. 1161/circulationaha.107.189420

Troussiere, A. C., Charley, C. M., Salleron, J., Richard, F., Delbeuck, X., Derambure, P., et al. (2014). Treatment of sleep apnoea syndrome decreases cognitive decline in patients with Alzheimer's disease. J. Neurol. Neurosurg. Psychiatry 85, 1405-1408. doi: 10.1136/jnnp-2013-307544

Tsapanou, A., Vlachos, G. S., Cosentino, S., Gu, Y., Manly, J. J., Brickman, A. M., et al. (2018). Sleep and subjective cognitive decline in cognitively healthy elderly: results from two cohorts. J. Sleep Res. 28:e12759. doi: 10.1111/jsr.12759

Wallace, A., and Bucks, R. S. (2013). Memory and obstructive sleep apnea: a meta-analysis. Sleep 36, 203-220. doi: 10.5665/sleep.2374

World Health Organization [WHO], (2014). C-reactive Protein Concentrations as a Marker of Inflammation or Infection for Interpreting Biomarkers of Micronutrient Status. Geneva: World Health Organization.

Yaffe, K., Laffan, A. M., Harrison, S. L., Redline, S., Spira, A. P., Ensrud, K. E., et al. (2011). Sleep-disordered breathing, hypoxia, and risk of mild cognitive impairment and dementia in older women. JAMA 306, 613-619. doi: 10.1001/ jama.2011.1115

Young, T., Palta, M., Dempsey, J., Skatrud, J., Weber, S., and Badr, S. (1993). The occurrence of sleep-disordered breathing among middle-aged adults. N. Engl. J. Med. 328, 1230-1235. doi: 10.1056/nejm199304293281704

Zhou, J., Camacho, M., Tang, X., and Kushida, C. A. (2016). A review of neurocognitive function and obstructive sleep apnea with or without daytime sleepiness. Sleep Med. 23, 99-108. doi: 10.1016/j.sleep.2016. 02.008

Zimmerman, M. E., and Aloia, M. S. (2012). Sleep-disordered breathing and cognition in older adults. Curr. Neurol. Neurosci. Rep. 12, 537-546. doi: 10.1007/ s11910-012-0298-z

\section{Conflict of Interest: MS and JV were employed by ReSurg SA.}

The remaining authors declare that the research was conducted in the absence of any commercial or financial relationships that could be construed as a potential conflict of interest.

Copyright (c) 2020 Attier-Zmudka, Sérot, Valluy, Saffarini, Douadi, Malinowski and Balédent. This is an open-access article distributed under the terms of the Creative Commons Attribution License (CC BY). The use, distribution or reproduction in other forums is permitted, provided the original author(s) and the copyright owner(s) are credited and that the original publication in this journal is cited, in accordance with accepted academic practice. No use, distribution or reproduction is permitted which does not comply with these terms. 